\title{
Novel insight into MALAT-1 in cancer: Therapeutic targets and clinical applications (Review)
}

\author{
DANYANG REN ${ }^{1}$, HUIYING LI ${ }^{1}$, RENQIU LI ${ }^{1}$, JIANMING SUN ${ }^{1}$, PIN GUO $^{1}$, \\ HUIYUN HAN ${ }^{1}$, YUEHUANG YANG ${ }^{2}$ and $\mathrm{JUN} \mathrm{LI}^{3}$ \\ ${ }^{1}$ Pharmaceutical Preparation Section and ${ }^{2}$ Department of Hematology, Children's Hospital of Kunming Medical University, \\ Kunming, Yunnan 650034; ${ }^{3}$ Department of Pharmacy, Anhui Medical University, Hefei, Anhui 230032, P.R. China
}

Received February 12, 2015; Accepted January 4, 2016

DOI: $10.3892 / \mathrm{ol} .2016 .4138$

\begin{abstract}
Long non-protein-coding RNAs (lncRNAs) are emerging as important gene expression regulators that are linked to various biological processes at the post-transcriptional and transcriptional levels. lncRNAs are known to be important in cell proliferation, cell differentiation, apoptosis and metastasis. Metastasis-associated lung adenocarcinoma transcript 1 (MALAT-1), a novel lncRNA, is highly conserved amongst mammals. In addition, it has been considered to act as an oncogene, depending on the tumor system. An increasing number of studies have indicated that MALAT-1 may be detected in certain types of human tumors, including lung and bladder cancer and hepatocellular carcinoma. MALAT-1 silencing may be an effective therapeutic approach against tumors. The present study reviews the current knowledge on the functional role of MALAT-1 in the control of various cancers.
\end{abstract}

\section{Contents}

1. Introduction

2. Overview of MALAT-1

3. Molecular targets of MALAT-1

4. MALAT-1 and epigenetic regulation

5. Potential clinical applications of MALAT-1

6. MALAT-1 and chemoresistance

7. Conclusion and future perspectives

Correspondence to: Professor Yuehuang Yang, Department of Hematology, Children's Hospital of Kunming Medical University, 288 Qianxing Road, Kunming, Yunnan 650034, P.R. China E-mail: etyyyangyuehuang@163.com

Professor Jun Li, Department of Pharmacy, Anhui Medical University, 81 Meishan Road, Hefei, Anhui 230032, P.R. China E-mail: 1ijun@ahmu.edu.cn

Key words: cancer, cancer therapy, metastasis associated lung adenocarcinoma transcript 1 , long non-protein-coding RNAs

\section{Introduction}

Previously, studies categorized non-coding (nc)RNAs as short ncRNA, mid-size ncRNA, and long ncRNA (lncRNA) by their lengths $(1,2)$. In addition, IncRNAs are subdivided by function, loci and post-transcriptional modification. IncRNAs have been operationally defined as transcripts that are produced by RNA polymerase II (Pol II), which are broadly classified as transcripts $>200$ nucleotides in length $(3,4)$. Transcriptome analyses have revealed that $70-90 \%$ of the mammalian genome was transcribed, but only 1-2\% may encode proteins (5). Additionally, lncRNAs that are localized to the nucleus possess stronger secondary structures. Therefore, knockdown of lncRNAs may not be sufficiently effective at evoking a phenotype and uncovering the physiological function of the lncRNA (5-8). The regulation of mRNA decay in the cytoplasm is crucial for controlling the abundance of cellular transcripts and the levels of protein expression. Therefore, the regulation of IncRNA decay in the nucleus is considered to be important for biological function (9). Overall, lncRNAs are important in the programming and regulation of the mammalian genome. The expression of lncRNAs is associated with numerous human diseases, including cancer (10).

The prevention of $20 \%$ of cancers in the United States alone would result in 300,000 fewer new cases annually (11). The growing knowledge of cancer treatment reveals methods to intercept cancers by novel, active approaches. Cancer results in high mortality and morbidity globally, largely due to the complex, heterogeneous nature of the disease and the lack of biomarkers for early diagnosis. A proteomics study of cancer identified functional proteins and drove the transformation of malignancy, identified biomarkers to detect early-stage cancer, determined therapy efficacy, identified novel drug targets and ultimately developed personalized medicine (12). Since tumor formation is a multistep process, normal cells evolve progressively to the neoplastic stage. Therefore, normal cells may acquire particular capacities that enable them to become tumorigenic. Over the last decade, remarkable progress was made in the field of cancer research, which led to a better understanding of cancer therapy (13).

Notably, metastasis associated lung adenocarcinoma transcript-1 (MALAT-1) is reported in numerous studies. For example, MALAT-1 was detected in the cerebellum of human 
Table I. The expression of MALAT-1 in cancer. MALAT-1 was expressed in all samples.

\begin{tabular}{lcc}
\hline Sample & Function in tumorigenesis & References \\
\hline HeLa, CaSki, SiHa and HCC94 cells & Oncogene & $(32,49)$ \\
Gallbladder carcinoma tissues & Oncogene & $(22)$ \\
Bladder cancer tissues & Oncogene & $(16,25)$ \\
Mesenchymal stem cells & Oncogene & $(75)$ \\
SGC-7901, MKN-45 and SUN-16 cells & Biomarker & $(5)$ \\
Human colorectal cancer tissues and LoVo and SW620 cells & Biomarker & $(20)$ \\
Liver cancer tissues and hepatocellular carcinoma cells & Biomarker & $(6,11)$ \\
Neuroblastoma SK-N-SH cells & Oncogene & $(75)$ \\
Non-small cell lung cancer cells & Biomarker & $(20,36)$ \\
Pancreatic ductal adenocarcinoma tissues & Biomarker & $(38)$ \\
Melanoma tissues & Oncogene & $(76)$ \\
Bladder urothelial carcinoma cells & Biomarker & $(3)$ \\
Brain metastasis lung cancer cells & Biomarker & $(10)$ \\
Colorectal cancer tissues & Biomarker & $(48)$ \\
Gastric cancer tissues & Biomarker & $(14)$ \\
\hline
\end{tabular}

MALAT-1, metastasis associated lung adenocarcinoma transcript-1.

alcoholics (14). However, MALAT-1 is a typical multifunctional gene that is important in a wide array of cancers, including bladder cancer $(15,16)$, gallbladder carcinoma (GBC) $(17,18)$, hepatocellular carcinoma (HCC) (19) and lung $(20,21)$ and gastric cancer (GC) (22). Therefore, understanding the regulation function of MALAT-1 within the context of cancer is of considerable significance. The present study focuses on the recent advances in the role of MALAT-1 in diverse cancers, as MALAT-1 possesses a typical multifunctional lncRNA (Table I).

\section{Overview of MALAT-1}

MALAT-1 is generally defined as a lncRNA that consists of $>8,000 \mathrm{nt}$ and is coded by chromosome 11q13. MALAT-1 transcripts are upregulated in a number of human carcinomas, highly expressed in numerous cancer types, including bladder cancer, gallbladder carcinoma, liver cancer, melanoma, colorectal cancer and gastric cancer, and associated with metastasis. MALAT-1 lacks a significant open reading frame; therefore, it may not translate proteins in vitro $(23,24)$. Studies have indicated that MALAT-1 possesses a distinct sequence or secondary structure that directs localization to nuclear speckles in human tumor cells $(25,26)$. Additionally, MALAT-1 expression may be abrogated using zinc finger nucleases (5). Notably, the quantitative loss of MALAT-1 did not affect proliferation, cell cycle progression or nuclear architecture in human lung or liver cancer cells (8). The MALAT-1 mouse model did not reveal any evident phenotype or histological abnormalities compared with wild-type animals. In addition, the loss of abundant nuclear MALAT-1 is compatible with cell viability and normal development (8). Previous studies have demonstrated that MALAT-1 interacts with pre-mRNA splicing factors, including the serine- and arginine-rich (SR) family of proteins (27-29). In detail, MALAT-1 may regulate numerous biological processes, including cancer cell migration, synapse formation, cell cycle progression and response to serum stimulation. However, MALAT-1 function becomes apparent only in specific cell types, such as metastatic cancer cells, and under particular conditions (27). MALAT-1 controls cell cycle progression by modulating the oncogenic transcription factor Myb-related protein B (B-MYB). B-MYB is a transcription factor that is required for the transcription of a large number of genes involved in mitotic progression (30).

$\mathrm{Xu}$ et al divided MALAT-1 into five fragments. The fragment located in the $3^{\prime}$ end $(6,918-8,441 \mathrm{nt})$, was pivotal in the biological processes of cell proliferation, migration and invasion in colorectal cancer (CRC) SW620 and SW480 cells (31). Notably, MALAT-1 exhibited substantially high expression levels in HeLa and MCF-7 cells and was clearly downregulated in a dose-dependent manner. According to the decline, MALAT-1 demonstrates a statistically significant dose-dependent decrease in human melanoma (BLM)-treated HeLa cells, but not in BLM-treated MCF-7 cells or irradiated cells (32). The 3 ' end of MALAT-1 is transcribed by Pol II and formed by the cleavage of ribonuclease $\mathrm{P}$ (RNase P). The 3 ' ends form a novel triple-helical structure that is essential for stimulating translation, the stability of the RNA and supporting export to the cytoplasm (33). Notably, another study indicated that the 3 ' end processing mechanism of MALAT-1 may yield a stable nuclear-retained ncRNA with a short poly (A) tail-like moiety and a small tRNA-like cytoplasmic RNA (34). MALAT-1 has been implicated in the regulation of mRNA splicing and expression (35). In addition, MALAT-1 is associated with prostate cancer progression, including castration-resistant prostate cancer (CRPC) (36). Tee et al observed that MALAT-1 induced neuroblastoma cell migration and invasion (37). MALAT-1 may also specifically regulate gene expression, but not alternative splicing in lung cancer cells (38). Additionally, MALAT-1 is abundantly expressed in the SK-N-SH cell line under 
normal culture conditions and the activation of the oxytocin receptor resulted in a significant increase of MALAT-1 expression (39). The results of an additional study of the 5' end of the MALAT-1 transcript indicated that an alternate transcription initiation site was used in the neuroblastoma cell line, which resulted in a shorter transcript compared with the previously reported transcript in lung cancer cells (39).

Previous studies have demonstrated that MALAT-1 is a highly conserved transcript that regulates the expression of metastasis-associated genes $(38,40)$. Analysis of the nuclear/cytoplasmic distribution of MALAT-1 during the cell cycle reveals a distinct profile, which demonstrates a profound enrichment in the G2/M phase in HeLa cells (28). The heterogeneous nuclear ribonucleoprotein (hnRNP) $\mathrm{C}$ protein is of particular interest, due to the RNA-binding capability of the protein and the reported cytoplasmic translocation in the G2/M phase (41). Compared with the insufficient binding capacity at the 5' end of the transcript, strong binding to the hnRNP C protein has been indicated in other regions of MALAT-1 (42). The function of MALAT-1 in the cell cycle may be regulated by facilitating the cytoplasmic translocation of the hnRNP C protein. However, in a previous study, MALAT-1 silencing only compromised the cytoplasmic translocation and not the expression of the hnRNP C protein (42).

\section{Molecular targets of MALAT-1}

At present, numerous studies have reported the antitumor role of MALAT-1 in cancer development (Table II). In addition, high expression of MALAT-1 has been indicated in cervical (30) and lung cancer (43). MALAT-1 is involved in the pathogenesis of cancers through the regulation of carcinoma-associated signaling pathways, including the mitogen-activated protein kinase (MAPK) signaling pathway (17). Tripathi et al showed that the specific deletion of MALAT-1 in human osteosarcoma cells led to the activation of p53 (30). MALAT-1-depleted cells demonstrated cell cycle defects that were sensitive to the p53 levels, which indicates that p53 is a main downstream mediator for MALAT-1 activity. In MALAT-1-depleted cells, replication of the S-phase was decreased and the cell population of G1 and G2/M cells was increased. In addition, MALAT-1 was involved in cervical cancer cell growth, cell cycle progression and invasion. The accumulation of evidence indicated that the downregulation of MALAT-1 may induce the expression of caspase-3, caspase-8 and B-cell lymphoma 2 (Bcl-2)-associated $X$ protein. In addition, MALAT-1 may suppress the expression of Bcl-2 and B-cell lymphoma-extra large. Overall, these results suggested that MALAT-1 may act as a therapeutic target in the prevention of human cervical cancer (44).

The knockdown of MALAT-1 in GBC cell lines may significantly inhibit the proliferation and metastasis of the GBC cells in vitro and in vivo. In addition, the extracellular signal-regulated kinase/MAPK signaling pathway may be inactivated in the GBC cell lines following MALAT-1 knockdown, which indicates that MALAT-1 may act as an oncogenic lncRNA that promotes the proliferation and metastasis of GBC (17). Other studies indicate that transforming growth factor- $\beta$ (TGF- $\beta$ ) induces MALAT-1 expression and epithelial-mesenchymal transition (EMT) in bladder cancer cells $(15,16,45,46)$. In addition, the inhibition of MALAT-1 or suppressor of zeste 12 suppressed the migratory and invasive properties induced by TGF- $\beta$ (15). A previous study revealed that the Wnt signaling pathway demonstrated particularly close links with EMT (47). Immunostaining analysis showed that MALAT-1-siRNA treatment significantly decreased the nuclear accumulation of $\beta$-catenin. Therefore, MALAT-1 promotes EMT and sequent cell migration by activating the Wnt/ $\beta$-catenin signaling pathway in bladder cancer cells (16).

MALAT-1 is recognized as an oncogene, as it regulates the alternative splicing of endogenous target genes that are involved in cancer (40). Taniguchi et al indicated that Myc-6 acts as a nuclear sequence-specific transcription factor, which specifically binds to the theoretical target site that is located within the 5'-upstream region of MALAT-1 (48). In addition, Myc- 6 exposure led to a dose-dependent decrease in the expression level of MALAT-1 in human osteosarcoma MG63 cells. Multiple serine/arginine-rich splicing factor 1 (SRSF1) proteins bound specifically and directly to the 5' end of MALAT-1. Additionally, MALAT-1 regulated alternative splicing by modulating the levels of active SR proteins (28). Li et al considered the potential significance that MALAT-1 promotes the activation effect of the key transcription factor Sp1 on the latent TGF- $\beta$-binding protein 3 (LTBP3) promoter by modulating the recruitment of Sp1 to the LTBP3 gene, which regulates the bioavailability of TGF- $\beta$, particularly in mesenchymal stem cells from myeloma patients (49). MALAT-1 may promote cell migration and decrease cell proliferation in CaSki cells. Notably, MALAT-1 may increase cell proliferation by upregulating cyclin D1, cyclin E and cluster of differentiation K6 (18). The study conducted by Wang et al indicated that MALAT-1 was overexpressed in GC cells (22). Additional studies revealed that MALAT-1 may induce a particularly high expression of SF2/ASF, an important member of the SR protein family, in the nucleolus. The depression of SF2/ASF induced poor cell proliferation in a similar way to MALAT-1 depletion; however, no significant effect on the cell proliferation depression or the loss of nuclear distribution of SF2/ASF was observed with low MALAT-1 expression. Therefore, MALAT-1 promotes cell proliferation in GC cells by recruiting SF2/ASF.

In tumorigenesis studies, MALAT-1 is one of several important splicing factor proline/glutamine-rich (SFPQ)-binding RNAs (50-53). SFPQs may also be termed PTB-associated splicing factors. Additionally, MALAT-1 may competitively bind to SFPQ to release SFPQ from the SFPQ/polypyrimidine tract binding protein 2 (PTBP2) complex in CRC LoVo cells. SFPQs, not PTBP2 proteins, are critical in the regulatory effect (54). In esophageal squamous cell carcinoma, MALAT-1 is suppressed by sex determining region Y (SRY)-box 17 via SRY binding-mediated transcriptional regulation (55).

Cancer metastasis has been indicated as the major cause of cancer recurrence and tumor-associated mortality. In a previous study, antisense oligonucleotides (ASOs) were designed to potently target MALAT-1. The altered ASOs effectively reduced MALAT-1 expression compared with the control ASO (38). A study conducted by Wang et al indicated an interaction between the Yes-associated protein (YAP) and SRSF1 by Angiomotin regulated MALAT-1 (19). The MALAT-1-mediated tumorigenesis in HCC provided a novel mechanism for YAP regulation of gene expression at 


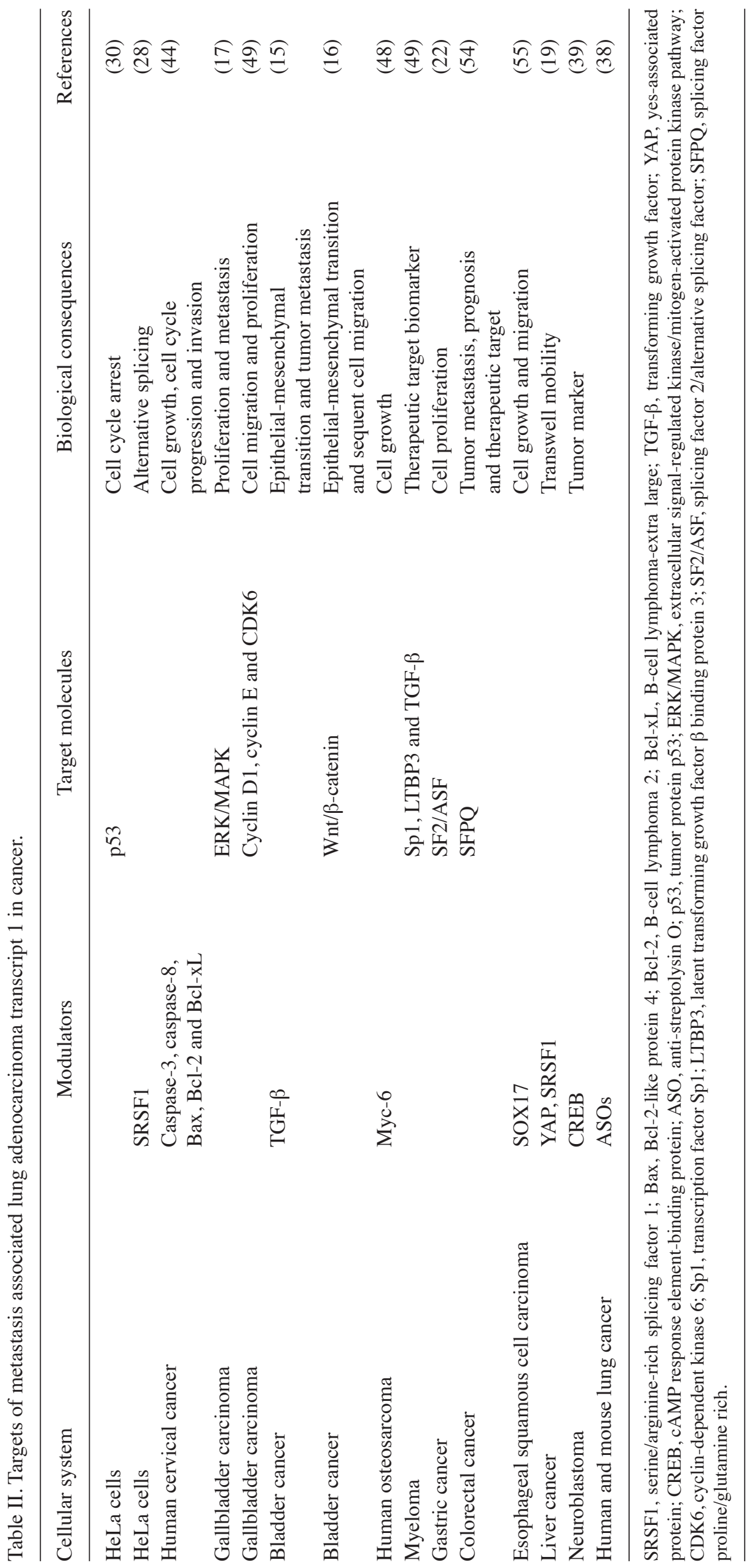




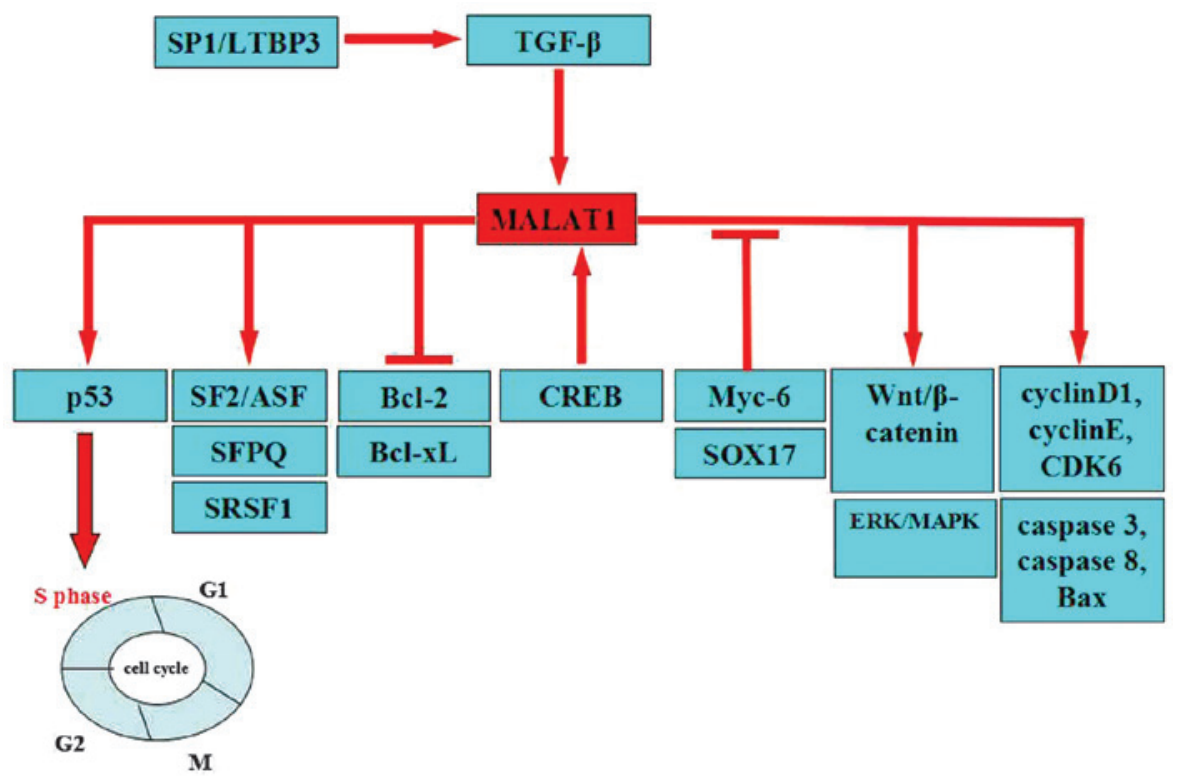

Figure 1. Target function of MALAT-1 in cancer. MALAT-1 acts as an oncogene by targeting significant tumor suppressor genes, including SF2/ASF, SFPQ, SRSF1, cyclin D1, cyclin E and CDK6. Overexpression of MALAT-1 increases cell proliferation through the activation of ERK/MAPK, p53, Wnt/3-catenin, caspase-3, caspase-8, the Bax signaling pathway and the cell cycle. MALAT-1 may suppress the expression of Bcl-2 and Bcl-xL. The expression of MALAT-1 is suppressed by Myc-6 and SOX17. TGF- $\beta$ induces MALAT-1 expression. CREB binds to the proximal promoter of the MALAT-1. MALAT-1, metastasis associated lung adenocarcinoma transcript 1; SF2/ASF, splicing factor 2/alternative splicing factor; SFPQ, splicing factor proline/glutamine rich; SRSF1, serine/arginine-rich splicing factor 1; CDK6, cyclin-dependent kinase 6; ERK/MAPK, extracellular signal-regulated kinase/mitogen-activated protein kinase pathway; Bax, Bcl-2-like protein 4; Bcl-2, B-cell lymphoma 2; Bcl-xL, B-cell lymphoma-extra large; TGF- $\beta$, transforming growth factor; CREB, cAMP response element-binding protein; Sox17, sex determining region Y-box 17; p53, tumor protein p53; Sp1/LTBP3, transcription factor Sp1/latent transforming growth factor $\beta$ binding protein 3 .

the transcriptional and post-transcriptional stages. The cyclic AMP-responsive element binding protein was indicated to bind to the proximal promoter of MALAT-1 in the human neuroblastoma SK-N-SH cell line (39). These results demonstrated that MALAT-1 expression may be modulated by the extracellular stimulation of tumor cells (Fig. 1).

The identification of MALAT-1 target genes that are involved in tumor development may aid the development of novel therapeutic strategies for tumor intervention.

\section{MALAT-1 and epigenetic regulation}

As aforementioned, the upregulation of MALAT-1 has been described in several types of human tumors. MALAT-1 silencing or gene therapy may be effective therapeutic approaches for human tumors $(10,38,56)$. At present, epigenetic mechanisms are important in the regulation of gene expression. The role of MALAT-1 is being elucidated in the epigenetic field. For example, post-translational histone modifications and RNA-based mechanisms, including those controlled by microRNAs, are significant mechanisms of the epigenetic regulation of gene expression. Overall, MALAT-1 may affect cancer development $(57,58)$. Notably, Jumonji C-domain-containing protein (JMJD)1A belongs to the JMJD family. Tee et al observed that the anti-JMJD1A antibody efficiently immunoprecipitated the MALAT-1 gene core promoter (37). In addtion, JMJD1A increased MALAT1 gene transcription by demethylating histone H3K9. Addtional studies revealed that JMJD1A increased MALAT-1 expression by directly binding to the MALAT-1 gene promoter, which lead to histone H3K9 demethylation by activating JMJD1A gene transcription $(59,60)$. Overall, the findings suggest that the development of more potent JMJD1A/MALAT-1 inhibitors may be used for the prevention of tumor metastasis (37). The methylation/demethylation cycle of Polycomb 2 (Pc2), a component of the polycomb repressive complex 1 , is responsible for the physical relocation of growth control genes from Polycomb bodies (PcGs). Methylated Pc2 may demonstrate an antimitogenic signal, but unmethylated Pc2 is essential for physiological growth control, gene expression and cell proliferation. Additionally, a sequence analysis of 94 various clones identified that taurine upregulated 1 (TUG1) repressed cell cycle genes, and was the most enriched RNA that interacted with methylated Pc2 (61). TUG1 localizes to PcGs and interacts with the methylated form of Pc2. However, MALAT-1 resides in nuclear speckles and only interacts with the unmethylated Pc2 protein (62). MALAT-1 silencing has been indicated to inhibit cell growth in HeLa cells, even in the presence of growth signals. The promoters that are associated with MALAT-1 in these domains exhibit high levels of activating the methylation of Lys36 at histone $\mathrm{H} 3$ trimethylation (H3K36me3) and H3K4me3 marks. This finding may be facilitated by the interaction of MALAT-1 with lysine demethylase 1 and SET nuclear proto-oncogene (SET) domain-containing 2. Histone methyltransferases and demethylases are associated with transcriptional activation and pre-mRNA splicing factors $(63,64)$. In summary, the results of previous studies may influence the chromatin modifications and accessibility of target gene loci.

Evidently, microRNA-125b (miR-125b), miR-99a and miR-100 are overexpressed in vincristine-resistant acute lymphoblastic leukemia(ALL).Co-expression of thesemiRNAs resulted in the downregulation ofDNAnucleotidylexotransferase, 


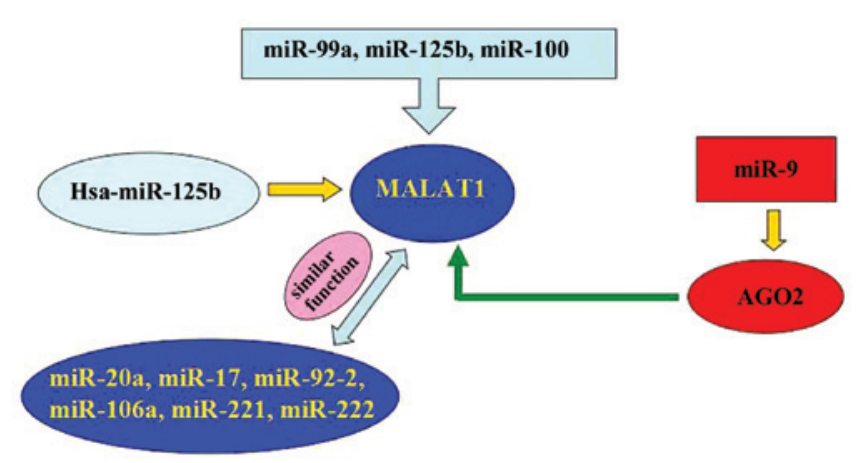

Figure 2. miR regulation of MALAT-1 in cancer. Co-expression of miR-125b, miR-99a, and miR-100 resulted in downregulation of MALAT-1. The function of miR-20a, miR-17, miR-92-2, miR-106a, miR-221 and miR-222 is similar to the function of MALAT-1 in mouse mammary tumors. miR-9 targets AGO2-mediated regulation of MALAT-1 and thus may regulate the expression of MALAT-1. Hsa-miR-125b mimic markedly inhibited MALAT-1 expression levels. MALAT-1, metastasis associated lung adenocarcinoma transcript 1; AGO2, argonaute-2; miR, microRNA.

nuclear casein kinase and cyclin-dependent kinase substrate 1, MALAT-1, small nuclear ribonucleoprotein polypeptide E, PNO1 protein, SET, kinesin-1 heavy chain, phosphoribosyl pyrophosphate synthetase 2, and ribosomal proteins S11, L38 and L23a. One study indicated that 7 of these genes, including MALAT-1, demonstrated decreased expression in the vincristine-resistant ALL cells of children (65). Another study showed that certain miRNAs, including miR-20a, -17, -92-2, -106a, -221 and -222, and chr19 ncRNA, which is similar to MALAT-1, may contribute to c-myc induced mouse mammary tumors (66). miR-9 may regulate the expression of MALAT-1. In addition, miR-9 may target AGO2-mediated regulation of MALAT-1 in the nucleus (67). Han et al hypothesized that Hsa-miR-125b is downregulated in bladder cancer compared with matched normal urothelium (68). However, sirtuin (SIRT)7 and MALAT-1 were upregulated in bladder cancer compared with matched normal urothelium. SIRT7 is identified as a crucial regulator of mitochondrial homeostasis. Additionally, high-grade and high-stage carcinomas demonstrated increased expression levels of SIRT7 and MALAT-1. However, additional studies indicated lower Hsa-miR-125b expression levels of SIRT7 and MALAT-1 compared with low-grade and low-stage carcinomas. In addition, Hsa-miR-125b mimics demonstrated markedly inhibited MALAT-1 expression levels in T24 and 5637 cells, whereas hsa-miR-125b inhibitors demonstrated markedly increased MALAT-1 expression levels. Notably, hsa-miR-125b binding sites within MALAT-1 were functional (Fig. 2).

\section{Potential clinical applications of MALAT-1}

Studies on MALAT-1 are, at present, at an early stage, and no extensive clinical studies on the expression of MALAT-1 in cancer tissues have been reported. In addition, the functional roles and associations of MALAT-1 with cancer are unclear. Therefore, MALAT-1 may be expected to potentially function as novel biomarkers in the diagnosis, prognosis, metastasis and the prediction of responses to therapy in solid tumors.

Numerous studies have assessed the biology of MALAT-1 in cancer (15-22). The role of MALAT-1 in cellular transformation suggests that MALAT-1 has the potential to function as a biomarker and a target for novel therapeutic approaches in multiple myeloma (MM). Previous studies have suggested that MALAT-1 expression in B-cell malignancies is decreased compared with solid tumors $(27,69)$. Another study demonstrated significantly lower MALAT-1 expression levels in the plasma of MM patients (70).

The study conducted by Jiang et al identified the correlation between MALAT-1 and human papillomavirus (HPV) (18). In this study, 64 cases of clinical cervical squamous cell carcinoma (SCC) samples were collected. MALAT-1 was identified in 6/18 cases in HPV-positive cervical normal cells and 14/22 cases in HPV-positive cervical lesion specimens. Therefore, these results suggested that HPV was an important factor that led to that activation of MALAT-1 in cervical SCC.

MALAT-1 was previously indicated to demonstrate extremely high expression levels in the established human non-small cell lung cancer (NSCLC) A549 and HTB58 cell lines. In addition, the downregulation of MALAT-1 decreased tumor growth in vivo. Human MALAT-1 transcripts increased the migration potential in the mouse fibroblast NIH3T3 cell line, and the downregulation of MALAT-1 decreased the migration potential and tumor growth in human NSCLC A549 cells in vivo. Overall, for SCC, increased expression of MALAT-1 may be associated with a poor prognosis (20). Notably, the A549 MALAT-1 wild-type cells and the two knockout (KO) cell lines with the lowest MALAT-1 expression, KO2 and KO3, were injected into the tail vein of nude mice, and the formation of the lung tumor nodules was analyzed 2 months later. The results of this pharmacological study suggested that MALAT-1 is required for effective tumor nodule formation in vivo, and therefore affects the metastatic process in lung cancer (38).

In order to identify tumor-associated MALAT-1 and to determine the correlation of the transcript with pancreatic duct adenocarcinoma (PDAC), Weber et al identified that MALAT-1 complies with the key characteristics of diagnostic biomarkers, including minimal invasiveness, high specificity and robustness (21). Alternatively, MALAT-1 may be applied as a complementary biomarker within a panel, in order to improve the entire diagnostic performance. The results of the study also showed that MALAT-1 expression levels are upregulated in pancreatic cancer tissues compared with adjacent noncancerous controls. Consistently, a higher expression level of MALAT-1 was identified in all seven pancreatic cancer cell lines relative to the human pancreatic ductal epithelial cell. Function analysis revealed that the downregulation of MALAT-1 may inhibit tumor cell proliferation and decrease cell migration and invasion. The underlying mechanisms are possibly involved in inducing G2/M cell cycle arrest, promoting cell apoptosis, suppressing EMT and reducing cancer stem-like properties. Therefore, the accumulation of evidence indicates that MALAT-1 may act as an oncogenic IncRNA that is involved in the malignant phenotype of pancreatic cancer. Importantly, MALAT-1 may be used as a potential therapeutic target (71). Another study indicated that the overexpression of MALAT-1, the tumor location and nerve invasion were independent predictors of disease-specific survival of PDAC (56).

MALAT-1 overexpression has been reported to predict the recurrence of HCC following liver transplantation (LT). MALAT-1 rs619586 was associated with a decreased HCC 
risk, with a borderline significance $(\mathrm{P}=0.057)$. Larger studies are required in order to clarify the associations between rs619586 in MALAT-1 and HCC risk (72). Notably, a consistently higher expression level of MALAT-1 was identified in all nine liver cancer cell lines, relative to the normal liver LO2 cell line. The association study indicated that the overexpression of MALAT-1 did not exhibit significant correlations with the pathological features of age, gender, tumor size, histological differentiation or portal vein tumor thrombi (73). More importantly, the multivariate regression analysis revealed that the overexpression of MALAT-1 may be a novel independent predictor of recurrence-free survival in HCC patients following LT. In addition, MALAT-1 silencing in HCC may be a potential anticancer therapy to prevent tumor recurrence following orthotopic liver transplantations (74). However, MALAT-1 is overexpressed in hepatoblastomas (HPBL) compared with HCC, as HCC and HPBL have distinct patterns of gene expression (75). Subsequent studies have provided evidence about the importance of MALAT-1 in liver cell proliferation, which was confirmed by the finding of arrested liver cell proliferation in response to partial MALAT-siRNA mediated knockdown $(40,76,77)$.

The expression level of MALAT-1 was significantly increased in melanoma tissues compared with paired adjacent normal tissues. Although no statistical difference of MALAT-1 expression was observed between melanomas with or without lymph node metastasis, the expression level of MALAT-1 was affected by the metastatic status of the tumor in melanoma. In addition, the migration of A-375 cells transfected with MALAT-1-siRNA was estimated using a Transwell assay. The cells with impaired expression of MALAT-1 migrated less effectively through the Transwell membrane. Therefore, the enhanced expression of MALAT-1 had the potential to affect cancer metastasis in melanomas. In general, these findings indicated that the expression level of MALAT-1 had the potential to be a prognostic indicator for the metastasis of melanoma (78).

$17 \beta$-Estradiol (E2) treatment to breast cell lines causes MALAT-1 RNA to decrease in an estrogen receptor $\alpha$ independent manner. This effect of E2 treatment is caused by MALAT-1 transcriptional regulation of E2. Zhao et al hypothesized that the effects of E2 treatment on breast cells are achieved by regulating MALAT-1 (79). However, MALAT-1 was downregulated in the cell culture, with the cells exhibiting high metastatic potential for ovarian cancer metastasis. The function of MALAT-1 may vary with various cancer types and context (80).

Several reports indicate that MALAT-1 contributes to the complex molecular mechanisms involved in the control of cell growth, differentiation and motility. Therefore, MALAT-1 may be important in the process of cancer metastasis. Han et al observed that MALAT-1 is upregulated in bladder urothelial carcinoma compared with the matched normal urothelium (81). A high expression level of MALAT-1 was associated with high-grade and high-stage bladder urothelial carcinoma. MALAT-1 silencing inhibited bladder urothelial carcinoma cell growth, induced apoptosis and decreased cell motility in T24 and 5637 cells. In addition, MALAT-1 knockdown also inhibited tumor metastasis (15). MALAT-1 inhibition may represent a promising therapeutic option for the suppression of bladder cancer progression. MALAT-1 expression levels were significantly upregulated in the majority of bladder cancer tissues compared with normal tissues. These data indicated that the upregulation of MALAT-1 may be associated with the onset of bladder cancer metastasis. In addition, MALAT-1 silencing impaired bladder cancer cell migration (16).

A study conducted by Okugawa et al illustrated that the expression levels of both MALAT-1 and HOX transcript antisense RNA were significantly increased in GC tissues compared with matching adjacent normal mucosa (82). Elevated MALAT-1 expression significantly correlated with peritoneal dissemination. Additionally, a difference in the levels of MALAT-1 was not evident in the plasma in a comparison between the healthy controls and GC patients (83).

The expression level of MALAT-1 was significantly increased in brain metastasis samples compared with non-brain metastasis samples. MALAT-1 was increased in a highly invasive subline of brain metastasis lung cancer cells. Functional studies indicated that MALAT-1 silencing inhibits a highly invasive subline of brain metastasis lung cancer cell migration and metastasis by inducing EMT (10).

A recent study indicated that the expression of MALAT-1 was higher in human CRC tissues than adjacent normal tissues (54). MALAT-1 upregulation has been demonstrated in CRC tumors. In addition, the expression levels of MALAT-1 in cancerous tissues were increased compared with noncancerous tissues. Although no associations were identified between MALAT-1 expression and the stages, including stage II and III, in CRC patients, the expression of MALAT-1 was significantly increased in male patients compared with female patients. In particular, patients with a high level of MALAT-1 expression showed significantly shorter disease-free survival (DFS) and overall survival times (OS) compared with patients with low MALAT-1 expression. In addition, patients with perineural invasion demonstrated significantly shorter DFS and OS times compared with those without perineural invasion. Multivariate Cox regression analysis indicated that MALAT-1 expression and perineural invasion were predictors of poor prognosis regarding DFS in CRC patients (84).

Another study investigated that MALAT-1 was upregulated across all 8 CRPC samples, which has been implicated in regulating mRNA splicing. The expression of MALAT-1 was also recently indicated to be associated with prostate cancer progression (36).

\section{MALAT-1 and chemoresistance}

MALAT-1 has been indicated to promote cell proliferation, migration and inhibit cell apoptosis in numerous cancer cells. The expression of MALAT-1 was reported to dramatically decrease with the increasing concentration of cisplatin and paclitaxel, which lengthened the treatment duration. Cisplatin and paclitaxel target significant lncRNAs in laryngeal squamous cell carcinoma (LSCC), which provides a novel molecular target to treat LSCC patients and sets direction for the development of novel drugs (85). Lung cancer is the top cause of cancer-associated mortality (86). One reason for this is the development of resistance to the chemotherapy treatment. In particular, cancer stem cells (CSCs) may escape treatment 
and regenerate the bulk of the tumor. Gene expression analysis showed that MALAT-1 was involved in tumor development and metastasis. In addition, MALAT-1 was similarly induced in CSCs and cisplatin-resistant H460 cells (87).

\section{Conclusion and future perspectives}

The identification of ncRNAs as important regulators of gene expression improved the understanding of numerous biological processes, including cancer cell migration, synapse formation, cell cycle progression and response to serum stimulation. The findings indicate that MALAT-1 is an important novel modulator in the development of cancers. MALAT-1 may promote cell proliferation, inhibit apoptosis, and enhance tumor growth in numerous cancer cell lines, and therefore, may serve as an excellent candidate for therapeutic intervention. High expression levels of MALAT-1 are widely hypothesized to associate with the prognosis and survival in clinical cancer patients. Notably, single MALAT-1 detection has strong potential as an independent prognostic factor in cancer patients. However, the applicability and epigenetic regulation of MALAT-1 targeted strategies for the clinical treatment of human tumors requires additional studies.

Additional studies are also required to elucidate the mechanism of MALAT-1, the emerging targets in oncology and the possible future directions for clinical applications. Therefore, in-depth studies on the functions of MALAT-1 may lead to novel diagnostic and therapeutic approaches.

\section{References}

1. Core LJ, Waterfall JJ and Lis JT: Nascent RNA sequencing reveals widespread pausing and divergent initiation at human promoters. Science 322: 1845-1848, 2008.

2. Taft RJ, Pang KC, Mercer TR, Dinger M and Mattick JS: Non-coding RNAs: Regulators of disease. J Pathol 220: 126-139, 2010.

3. Gutschner T, Hämmerle M and Diederichs S: MALAT1 - a paradigm for long noncoding RNA function in cancer. $\mathrm{J}$ Mol Med Berl 91: 791-801, 2013.

4. Qiu MT, Hu JW, Yin R and Xu L: Long noncoding RNA: An emerging paradigm of cancer research. Tumour Biol 34: 613-620, 2013.

5. Gutschner T, Baas M and Diederichs S: Noncoding RNA gene silencing through genomic integration of RNA destabilizing elements using zinc finger nucleases. Genome Res 21: 1944-1954, 2011.

6. Zhang B, Arun G, Mao YS, Lazar Z, Hung G, Bhattacharjee G, Xiao X, Booth CJ, Wu J, Zhang C and Spector DL: The lncRNA Malat1 is dispensable for mouse development but its transcription plays a cis-regulatory role in the adult. Cell Rep 2: 111-123, 2012.

7. Gutschner T and Diederichs S: The hallmarks of cancer: A long non-coding RNA point of view. RNA Biol 9: 703-719, 2012.

8. Eißmann M, Gutschner T, Hämmerle M, Günther $S$, Caudron-Herger M, Groß M, Schirmacher P, Rippe K, Braun T, Zörnig $\mathrm{M}$ and Diederichs S: Loss of the abundant nuclear non-coding RNA MALAT1 is compatible with life and development. RNA Biol 9: 1076-1087, 2012.

9. Tani H, Nakamura Y, Ijiri K and Akimitsu N: Stability of MALAT-1, a nuclear long non-coding RNA in mammalian cells, varies in various cancer cells. Drug Discov Ther 4: 235-239, 2010.

10. Shen L, Chen L, Wang Y, Jiang X, Xia H and Zhuang Z: Long noncoding RNA MALAT1 promotes brain metastasis by inducing epithelial-mesenchymal transition in lung cancer. J Neurooncol 121: 101-108, 2015

11. Perera FP and Vineis P: Cancer. IARC Sci Publ: 337-362, 2011.

12. Tan HT, Lee YH and Chung MC: Cancer proteomics. Mass Spectrom Rev 31: 583-605, 2012.
13. Dillman RO: Cancer immunotherapy. Cancer Biother Radiopharm 26: 1-64, 2011

14. Kryger R, Fan L, Wilce PA and Jaquet V: MALAT-1, a non protein-coding RNA is upregulated in the cerebellum, hippocampus and brain stem of human alcoholics. Alcohol 46: 629-634, 2012.

15. Fan Y, Shen B, Tan M, Mu X, Qin Y, Zhang F and Liu Y: TGF- $\beta$-induced upregulation of malat 1 promotes bladder cancer metastasis by associating with suz12. Clin Cancer Res 20: 1531-1541, 2014.

16. Ying L, Chen Q, Wang Y, Zhou Z, Huang Y and Qiu F: Upregulated MALAT-1 contributes to bladder cancer cell migration by inducing epithelial-to-mesenchymal transition. Mol Biosyst 8: 2289-2294, 2012.

17. Wu XS, Wang XA, Wu WG, Hu YP, Li ML, Ding Q, Weng H, Shu YJ, Liu TY, Jiang L, et al: MALAT1 promotes the proliferation and metastasis of gallbladder cancer cells by activating the ERK/MAPK pathway. Cancer Biol Ther 15: 806-814, 2014.

18. Jiang Y, Li Y, Fang S, Jiang B, Qin C, Xie P, Zhou G and Li G: The role of MALAT1 correlates with HPV in cervical cancer. Oncol Lett 7: 2135-2141, 2014.

19. Wang J, Wang H, Zhang Y, Zhen N, Zhang L, Qiao Y, Weng W, Liu X, Ma L, Xiao W, et al: Mutual inhibition between YAP and SRSF1 maintains long non-coding RNA, Malat1-induced tumourigenesis in liver cancer. Cell Signal 26: 1048-1059, 2014.

20. Schmidt LH, Spieker T, Koschmieder S, Schäffers S, Humberg J, Jungen D, Bulk E, Hascher A, Wittmer D, Marra A, et al: The long noncoding MALAT-1 RNA indicates a poor prognosis in non-small cell lung cancer and induces migration and tumor growth. J Thorac Oncol 6: 1984-1992, 2011.

21. Weber DG, Johnen G, Casjens S, Bryk O, Pesch B, Jöckel KH, Kollmeier $J$ and Brüning T: Evaluation of long noncoding RNA MALAT1 as a candidate blood-based biomarker for the diagnosis of non-small cell lung cancer. BMC Res Notes 6: 518, 2013.

22. Wang J, Su L, Chen X, Li P, Cai Q, Yu B, Liu B, Wu W and Zhu Z: MALAT1 promotes cell proliferation in gastric cancer by recruiting SF2/ASF. Biomed Pharmacother 68: 557-564, 2014.

23. Ji P, Diederichs S, Wang W, Böing S, Metzger R, Schneider PM, Tidow N, Brandt B, Buerger H, Bulk E, et al: MALAT-1, a novel noncoding RNA, and thymosin beta4 predict metastasis and survival in early-stage non-small cell lung cancer. Oncogene 22: 8031-8041, 2003.

24. Rajaram V, Knezevich S, Bove KE, Perry A and Pfeifer JD: DNA sequence of the translocation breakpoints in undifferentiated embryonal sarcoma arising in mesenchymal hamartoma of the liver harboring the $\mathrm{t}(11 ; 19)$ (q11;q13.4) translocation. Genes Chromosomes Cancer 46: 508-513, 2007.

25. Miyagawa R, Tano K, Mizuno R, Nakamura Y, Ijiri K, Rakwal R, Shibato J, Masuo Y, Mayeda A, Hirose T and Akimitsu N: Identification of cis- and trans-acting factors involved in the localization of MALAT-1 noncoding RNA to nuclear speckles. RNA 18: 738-751, 2012.

26. Hutchinson JN, Ensminger AW, Clemson CM, Lynch CR, Lawrence JB and Chess A: A screen for nuclear transcripts identifies two linked noncoding RNAs associated with SC35 splicing domains. BMC Genomics 8: 39, 2007.

27. Nakagawa S, Ip JY, Shioi G, Tripathi V, Zong X, Hirose T and Prasanth KV: Malat1 is not an essential component of nuclear speckles in mice. RNA 18: 1487-1499, 2012.

28. Tripathi V, Ellis JD, Shen Z, Song DY, Pan Q, Watt AT, Freier SM, Bennett CF, Sharma A, Bubulya PA, et al: The nuclear-retained noncoding RNA MALAT1 regulates alternative splicing by modulating SR splicing factor phosphorylation. Mol Cell 39: 925-938, 2010.

29. Spector DL and Lamond AI: Nuclear speckles. Cold Spring Harb Perspect Biol 3: a000646, 2011.

30. Tripathi V, Shen Z, Chakraborty A, Giri S, Freier SM, Wu X, Zhang Y, Gorospe M, Prasanth SG, Lal A and Prasanth KV: Long noncoding RNA MALAT1 controls cell cycle progression by regulating the expression of oncogenic transcription factor B-MYB. PLoS Genet 9: e1003368, 2013.

31. Xu C, Yang M, Tian J, Wang X and Li Z: MALAT-1: A long non-coding RNA and its important 3 ' end functional motif in colorectal cancer metastasis. Int J Oncol 39: 169-175, 2011.

32. Özgür E, Mert U, Isin M, Okutan M, Dalay N and Gezer U: Differential expression of long non-coding RNAs during genotoxic stress-induced apoptosis in HeLa and MCF-7 cells. Clin Exp Med 13: 119-126, 2013.

33. Marzluff WF: Novel 3' ends that support translation. Genes Dev 26: 2457-2460, 2012. 
34. Wilusz JE, Freier SM and Spector DL: 3' end processing of a long nuclear-retained noncoding RNA yields a tRNA-like cytoplasmic RNA. Cell 135: 919-932, 2008.

35. Tollervey JR, Curk T, Rogelj B, Briese M, Cereda M, Kayikci M, Hortobágyi T, Nishimura AL, Zupunski V, et al: Characterising the RNA targets and position-dependent splicing regulation by TDP-43. Nat Neurosci 14: 452-458, 2011.

36. Sowalsky AG, Xia Z, Wang L, Zhao H, Chen S, Bubley GJ, Balk SP and Li W: Whole transcriptome sequencing reveals extensive unspliced mRNA in metastatic castration-resistant prostate cancer. Mol Cancer Res 13: 98-106, 2015

37. Tee AE, Ling D, Nelson C, Atmadibrata B, Dinger ME, Xu N, Mizukami T, Liu PY, Liu B, Cheung B, et al: The histone demethylase JMJD1A induces cell migration and invasion by up-regulating the expression of the long noncoding RNA MALAT1. Oncotarget 5: 1793-1804, 2014.

38. Gutschner T, Hämmerle M, Eissmann M, Hsu J, Kim Y, Hung G, Revenko A, Arun G, Stentrup M, Gross M, et al: The noncoding RNA MALAT1 is a critical regulator of the metastasis phenotype of lung cancer cells. Cancer Res 73: 1180-1189, 2013

39. Koshimizu TA, Fujiwara Y, Sakai N, Shibata K and Tsuchiya H: Oxytocin stimulates expression of a noncoding RNA tumor marker in a human neuroblastoma cell line. Life Sci 86 455-460, 2010

40. Mohamadkhani A: Long noncoding RNAs in interaction with RNA binding proteins in hepatocellular carcinoma. Hepat Mon 14: e18794, 2014.

41. Schepens B, Tinton SA, Bruynooghe Y, Parthoens E, Haegman M, Beyaert R and Cornelis S: A role for hnRNP $\mathrm{C} 1 / \mathrm{C} 2$ and Unr in internal initiation of translation during mitosis. EMBO J 26: 158-169, 2007.

42. Yang F, Yi F, Han X, Du Q and Liang Z: MALAT-1 interacts with hnRNP $C$ in cell cycle regulation. FEBS Lett 587: 3175-3181, 2013

43. Guffanti A, Iacono M, Pelucchi P, Kim N, Soldà G, Croft LJ, Taft RJ, Rizzi E, Askarian-Amiri M, Bonnal RJ, et al: A transcriptional sketch of a primary human breast cancer by 454 deep sequencing. BMC Genomics 10: 163, 2009.

44. Guo F, Li Y, Liu Y, Wang J, Li Y and Li G: Inhibition of metastasis-associated lung adenocarcinoma transcript 1 in CaSki human cervical cancer cells suppresses cell proliferation and invasion. Acta Biochim Biophys Sin (Shanghai) 42: 224-229, 2010

45. Wei H, Kamat AM, Aldousari S, Ye Y, Huang M, Dinney CP and $\mathrm{Wu} \mathrm{X}$ : Genetic variations in the transforming growth factor beta pathway as predictors of bladder cancer risk. PLoS One 7 : e51758, 2012.

46. Al-Azayzih A, Gao F, Goc A and Somanath PR: TGF $\beta 1$ induces apoptosis in invasive prostate cancer and bladder cancer cells via Akt-independent, p38 MAPK and JNK/SAPK-mediated activation of caspases. Biochem Biophys Res Commun 427: 165-170, 2012.

47. Xu W, Ji J, Xu Y, Liu Y, Shi L, Liu Y, Lu X, Zhao Y, Luo F, Wang B, et al: MicroRNA-191, by promoting the EMT and increasing CSC-like properties, is involved in neoplastic and metastatic properties of transformed human bronchial epithelial cells. Mol Carcinog 54 (Suppl 1): E148-E161, 2015.

48. Taniguchi M, Fujiwara K, Nakai Y, Ozaki T, Koshikawa N, Toshio K, Kataba M, Oguni A, Matsuda H, Yoshida Y, et al: Inhibition of malignant phenotypes of human osteosarcoma cells by a gene silencer, a pyrrole-imidazole polyamide, which targets an E-box motif. FEBS Open Bio 4: 328-334, 2014.

49. Li B, Chen P, Qu J, Shi L, Zhuang W, Fu J, Li J, Zhang X, Sun Y and Zhuang W: Activation of LTBP3 gene by a long noncoding RNA (lncRNA) MALAT1 transcript in mesenchymal stem cells from multiple myeloma. J Biol Chem 289: 29365-29375, 2014.

50. Li L, Feng TT, Lian YY, Zhang GF, Garen A and Song X: Role of human noncoding RNAs in the control of tumorigenesis. Proc Natl Acad Sci USA 106: 12956-12961, 2009.

51. Wang G, Cui Y, Zhang G, Garen A and Song X: Regulation of proto-oncogene transcription, cell proliferation, and tumorigenesis in mice by PSF protein and a VL30 noncoding RNA. Proc Natl Acad Sci USA 106: 16794-16798, 2009.

52. Sephton CF, Cenik C, Kucukural A, Dammer EB, Cenik B, Han Y, Dewey CM, Roth FP, Herz J, Peng J, et al: Identification of neuronal RNA targets of TDP-43-containing ribonucleoprotein complexes. J Biol Chem 286: 1204-1215, 2011.

53. Rajesh C, Baker DK, Pierce AJ and Pittman DL: The splicing-factor related protein SFPQ/PSF interacts with RAD51D and is necessary for homology-directed repair and sister chromatid cohesion. Nucleic Acids Res 39: 132-145, 2011
54. Ji Q, Zhang L, Liu X, Zhou L, Wang W, Han Z, Sui H, Tang Y, Wang Y, Liu N, et al: Long non-coding RNA MALAT1 promotes tumour growth and metastasis in colorectal cancer through binding to SFPQ and releasing oncogene PTBP2 from SFPQ/PTBP2 complex. Br J Cancer 111: 736-748, 2014

55. Kuo IY, Wu CC, Chang JM, Huang YL, Lin CH, Yan JJ, Sheu BS, Lu PJ, Chang WL, Lai WW and Wang YC: Low SOX17 expression is a prognostic factor and drives transcriptional dysregulation and esophageal cancer progression. Int J Cancer 135: 563-573, 2014

56. Liu JH, Chen G, Dang YW, Li CJ and Luo DZ: Expression and prognostic significance of lncRNA MALAT1 in pancreatic cancer tissues. Asian Pac J Cancer Prev 15: 2971-2977, 2014.

57. Abi Khalil C: The emerging role of epigenetics in cardiovascular disease. Ther Adv Chronic Dis 5: 178-187, 2014.

58. Asrih $\mathrm{M}$ and Steffens S: Emerging role of epigenetics and miRNA in diabetic cardiomyopathy. Cardiovasc Pathol 22: 117-125, 2013.

59. Park SJ, Kim JG, Son TG, Yi JM, Kim ND, Yang K and Heo K: The histone demethylase JMJD1A regulates adrenomedullin-mediated cell proliferation in hepatocellular carcinoma under hypoxia. Biochem Biophys Res Commun 434: 722-727, 2013.

60. Osawa T, Tsuchida R, Muramatsu M, Shimamura T, Wang F, Suehiro J, Kanki Y, Wada Y, Yuasa Y, Aburatani H, et al: Inhibition of histone demethylase JMJD1A improves anti-angiogenic therapy and reduces tumor-associated macrophages. Cancer Res 73: 3019-3028, 2013.

61. Yang L, Lin C, Liu W, Zhang J, Ohgi KA, Grinstein JD, Dorrestein PC and Rosenfeld MG: NcRNA- and Pc2 methylation-dependent gene relocation between nuclear structures mediates gene activation programs. Cell 147: 773-788, 2011.

62. Smith MA, Gesell T, Stadler PF and Mattick JS: Widespread purifying selection on RNA structure in mammals. Nucleic Acids Res 41: 8220-8236, 2013

63. Hu Q, Kwon YS, Nunez E, Cardamone MD, Hutt KR, Ohgi KA, Garcia-Bassets I, Rose DW, Glass CK, Rosenfeld MG and Fu XD: Enhancing nuclear receptor-induced transcription requires nuclear motor and LSD1-dependent gene networking in interchromatin granules, Proc Natl Acad Sci USA 105: 19199-19204, 2008.

64. Wagner EJ and Carpenter PB: Understanding the language of Lys36 methylation at histone H3. Nat Rev Mol Cell Biol 13: $115-126,2012$.

65. Akbari Moqadam F, Lange-Turenhout EA, Ariës IM, Pieters R and den Boer ML: MiR-125b, miR-100 and miR-99a co-regulate vincristine resistance in childhood acute lymphoblastic leukemia. Leuk Res 37: 1315-1321, 2013.

66. Sun Y, Wu J, Wu SH, Thakur A, Bollig A, Huang Y and Liao DJ: Expression profile of microRNAs in c-Myc induced mouse mammary tumors. Breast Cancer Res Treat 118: 185-196, 2009.

67. Leucci E, Patella F, Waage J, Holmstrøm K, Lindow M, Porse B, Kauppinen S and Lund AH: MicroRNA-9 targets the long non-coding RNA MALAT1 for degradation in the nucleus. Sci Rep 3: 2535, 2013.

68. Han Y, Liu Y, Zhang H, Wang T, Diao R, Jiang Z, Gui Y and Cai Z: Hsa-miR-125b suppresses bladder cancer development by down-regulating oncogene SIRT7 and oncogenic long non-coding RNA MALAT1. FEBS Lett 587: 3875-3882, 2013.

69. Bernard D, Prasanth KV, Tripathi V, Colasse S, Nakamura T, Xuan Z, Zhang MQ, Sedel F, Jourdren L, Coulpier F, et al: A long nuclearretained non-coding RNA regulates synaptogenesis by modulating gene expression. EMBO J 29: 3082-3093, 2010.

70. Isin M, Ozgur E, Cetin G, Erten N, Aktan M, Gezer U and Dalay N: Investigation of circulating lncRNAs in B-cell neoplasms. Clin Chim Acta 431: 255-259, 2014.

71. Bonasio R and Shiekhattar R: Regulation of transcription by long noncoding RNAs. Annu Rev Genet 48: 433-455, 2014.

72. Liu Y, Pan S, Liu L, Zhai X, Liu J, Wen J, Zhang Y, Chen J, Shen $H$ and $\mathrm{Hu} \mathrm{Z}$ : A genetic variant in long non-coding RNA HULC contributes to risk of HBV-related hepatocellular carcinoma in a Chinese population. PLoS One 7: e35145, 2012.

73. Li G, Zhang H, Wan X, Yang X, Zhu C, Wang A, He L, Miao R, Chen $\mathrm{S}$ and Zhao $\mathrm{H}$ : Long noncoding RNA plays a key role in metastasis and prognosis of hepatocellular carcinoma. Biomed Res Int 2014: 780521, 2014.

74. Lai MC, Yang Z, Zhou L, Zhu QQ, Xie HY, Zhang F, Wu LM, Chen LM and Zheng SS: Long non-coding RNA MALAT-1 overexpression predicts tumor recurrence of hepatocellular carcinoma after liver transplantation. Med Oncol 29: 1810-1816, 2012.

75. Luo JH, Ren B, Keryanov S, Tseng GC, Rao UN, Monga SP, Strom S, Demetris AJ, Nalesnik M, Yu YP, et al: Transcriptomic and genomic analysis of human hepatocellular carcinomas and hepatoblastomas. Hepatology 44: 1012-1024, 2006. 
76. Watts R, Ghozlan M, Hughey CC, Johnsen VL, Shearer J and Hittel DS: Myostatin inhibits proliferation and insulin-stimulated glucose uptake in mouse liver cells. Biochem Cell Biol 92: 226-234, 2014.

77. He Y, Meng XM, Huang C, Wu BM, Zhang L, Lv XW and Li J: Long noncoding RNAs: Novel insights into hepatocelluar carcinoma. Cancer Lett 344: 20-27, 2014.

78. Tian Y, Zhang X, Hao Y, Fang Z and He Y: Potential roles of abnormally expressed long noncoding RNA UCA1 and MALAT-1 in metastasis of melanoma. Melanoma Res 24: 335-341, 2014.

79. Zhao Z, Chen C, Liu Y and Wu C: $17 \beta$-Estradiol treatment inhibits breast cell proliferation, migration and invasion by decreasing MALAT-1 RNA level. Biochem Biophys Res Commun 445: 388-393, 2014.

80. Liu SP, Yang JX, Cao DY and Shen K: Identification of differentially expressed long non-coding RNAs in human ovarian cancer cells with different metastatic potentials. Cancer Biol Med 10: 138-141, 2013.

81. Han Y, Liu Y, Nie L, Gui Y and Cai Z: Inducing cell proliferation inhibition, apoptosis, and motility reduction by silencing long noncoding ribonucleic acid metastasis-associated lung adenocarcinoma transcript 1 in urothelial carcinoma of the bladder. Urology 81: 209e1-209.e7, 2013.

82. Okugawa Y, Toiyama Y, Hur K, Toden S, Saigusa S, Tanaka K, Inoue Y, Mohri Y, Kusunoki M, Boland CR and Goel A: Metastasis-associated long non-coding RNA drives gastric cancer development and promotes peritoneal metastasis Carcinogenesis 35: 2731-2739, 2014.
83. Arita T, Ichikawa D, Konishi H, Komatsu S, Shiozaki A, Shoda K, Kawaguchi T, Hirajima S, Nagata H, Kubota T, et al: Circulating long non-coding RNAs in plasma of patients with gastric cancer. Anticancer Res 33: 3185-3193, 2013.

84. Zheng HT, Shi DB, Wang YW, Li XX, Xu Y, Tripathi P, Gu WL, Cai GX and Cai SJ: High expression of lncRNA MALAT1 suggests a biomarker of poor prognosis in colorectal cancer. Int J Clin Exp Pathol 7: 3174-3181, 2014.

85. Chen H, Xin Y, Zhou L, Huang JM, Tao L, Cheng L and Tian $\mathrm{J}$ : Cisplatin and paclitaxel target significant long noncoding RNAs in laryngeal squamous cell carcinoma. Med Oncol 31: 246, 2014.

86. Hong SH, Park SJ, Lee S, Cho CS and Cho MH: Aerosol gene delivery using viral vectors and cationic carriers for in vivo lung cancer therapy. Expert Opin Drug Deliv 12: 977-991, 2015.

87. Lopez-Ayllon BD, Moncho-Amor V, Abarrategi A, Ibañez de Cáceres I, Castro-Carpeño J, Belda-Iniesta C, Perona R and Sastre L: Cancer stem cells and cisplatin-resistant cells isolated from non-small-lung cancer cell lines constitute related cell populations. Cancer Med 3: 1099-1111, 2014. 